\title{
Displacement of Boron from the Silicon Crystal Nodes by Interstitial Si Atoms During Implantation and Annealing
}

\author{
${ }^{1}$ M. Jadan, ${ }^{2}$ A.R. Chelyadinskii and ${ }^{2}$ V. Yu Yavid \\ ${ }^{1}$ Tafila Applied University College, AL-Balqa' Applied University, Jordan \\ ${ }^{2}$ Belarusian State University, Minsk, Belarus
}

\begin{abstract}
The process of boron displacement from the nodes into interstitial positions of interstitial $\mathrm{Si}$ atoms in silicon (Watkins effect) on the conditions of implantation and annealing has been investigated with the help of X-ray diffraction and electrical methods. It was revealed that the efficiency of the Watkins substitution is determined by the ion current density (level of ionization). With increasing of the ionization level in the implanted layer during implantation or annealing (additional low-energy electron irradiation) the replacement process may be suppressed.
\end{abstract}

Key words: Silicon, Ion Implantation, Radiation Defects, Watkins Substitution

\section{INTRODUCTION}

Radiation defects are generated in silicon during ion implantation. In the process of subsequent thermal treatment to anneal the defects and activate the inserted impurities, residual extended damages (rod-like defects, stacking faults bounded by dislocation loops) may be formed. These extended damages have interstitial nature. The presence of such impurities as $\mathrm{C}$ or $\mathrm{B}$ in the silicon crystals (layers) suppresses the formation of residual damages ${ }^{[1-3]}$. The necessary condition for a positive result is the incorporation of these impurities in the lattice nodes. But it is known that during implantation the quantity of generating interstitial $\mathrm{Si}$ atoms exceeds the value for stable interstitial complexes by a factor of roughly 20 . The selfinterstitials may displace these impurities from the lattice nodes (Watkins substitution effect ${ }^{[4]}$ ). Therefore it is needed to preserve these impurities in the lattice nodes during implantation, so that they can later operate as traps for self-interstitials during the heat treatment. In the case of boron it is important for multiple step implantation $^{[5]}$, for example. Using of boron atoms as the traps for self-interstitial must decrease the amount of the operations in the multiple step method of ion doping. In this work we investigate the process of boron displacement from the nodes into interstitial positions by interstitial $\mathrm{Si}$ atoms on the conditions of implantation and annealing.

\section{MATERIALS AND METHODS}

In this work the change of the crystal lattice period $\Delta \mathrm{a}$ was investigated in implanted silicon layers. The $\Delta \mathrm{a}$ values were measured by the angle difference between diffraction maxima of the X-ray reflection from the implanted layer and the substrate. The diffraction curves were measured by a double crystal X-ray spectrometer for a parallel position of the crystalmonochromator with the sample using $\mathrm{CuK}_{\alpha 1}$ irradiation in the fourth order of a (111) reflection. The accuracy of the $\Delta \mathrm{a}$ determination was $\pm 1 \times 10^{-6} \mathrm{~nm}$. Silicon crystals lightly doped with phosphorus $\left(\rho_{0}=0.5\right.$ $\Omega \mathrm{cm})$ and highly doped with boron $\left(\rho_{0}=0.005 \Omega \mathrm{cm}\right)$ were implanted with a scanning beam of $100 \mathrm{keV} \mathrm{B}^{+}$ ions with the effective current densities $j_{\mathrm{ef}}=0.05-0.5$ $\mu \mathrm{A} \mathrm{cm}^{-2}$.

The charge carrier concentration was determined from the Hall effect measurements in the Van der Pauw configuration ${ }^{[6]}$.

Isochronal $(15 \mathrm{~min})$ annealing of the implanted samples was carried out in a vacuum chamber both without and with simultaneous $10 \mathrm{keV}$ electron irradiation with current density of $5 \mu \mathrm{A} \mathrm{cm}$. The accuracy of temperature during annealing was $\pm 2{ }^{\circ} \mathrm{C}$.

\section{RESULTS AND DISCUSSION}

In Fig. 1 the change (growth) of the lattice constant $\Delta \mathrm{a}$ versus the $\mathrm{B}^{+}$ion fluence $\Phi$ is given for silicon samples lightly phosphorus doped (curve 1) and heavily doped with boron (curves 2, 3,4), respectively. Curves $\Delta \mathrm{a}(\Phi)$ are noticeably higher for silicon heavily doped with boron $\left(\rho_{0}=0.005 \Omega \mathrm{cm}\right)$ than those of the n-type silicon samples. It should be noted that the values of $\Delta \mathrm{a}$ $(\Phi)$ are higher for a lower ion current density.

Figure 2 demonstrates the recovery of the lattice constant in silicon layers implanted with $\mathrm{B}^{+}$ions during isochronal annealing. In n-type silicon $\left(\rho_{o}=0.5 \Omega \mathrm{cm}\right)$ on the first stage $\left(100-280^{\circ} \mathrm{C}\right)$ the deficiencies are annealed mainly ${ }^{[7]}$. At the second stage $\left(380-600^{\circ} \mathrm{C}\right)$ the multivacancy complexes, e.g. Five-vacancy (Si-P1 centers) and others, which remained unidentified ${ }^{[8]}$ are annealed. The same two stages were revealed for silicon implanted with $\mathrm{S}^{+}$ions ${ }^{[9]}$. 


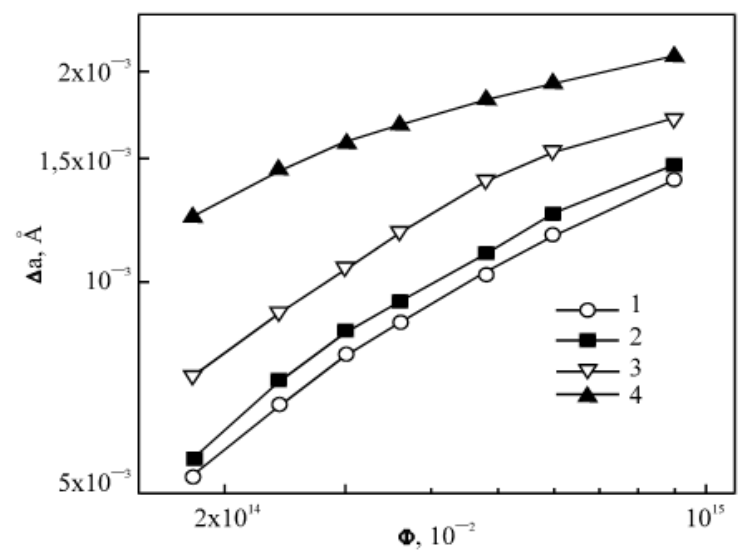

Fig. 1: Dependence of the Silicon Lattice Constant $\Delta \mathrm{a}$ on $\mathrm{B}^{+}$Ion Fluence $\Phi$ in:1)-Si:P, $\rho_{0}=0.5 \Omega \mathrm{cm}$; (2-4)-Si:B, $\rho_{0}=0.005 \Omega \mathrm{cm}$; jef $:(1,3)-0.2 ;(2)$ - 0.5; (4) $-0.05 \mu \mathrm{A} \mathrm{cm}^{-2}$

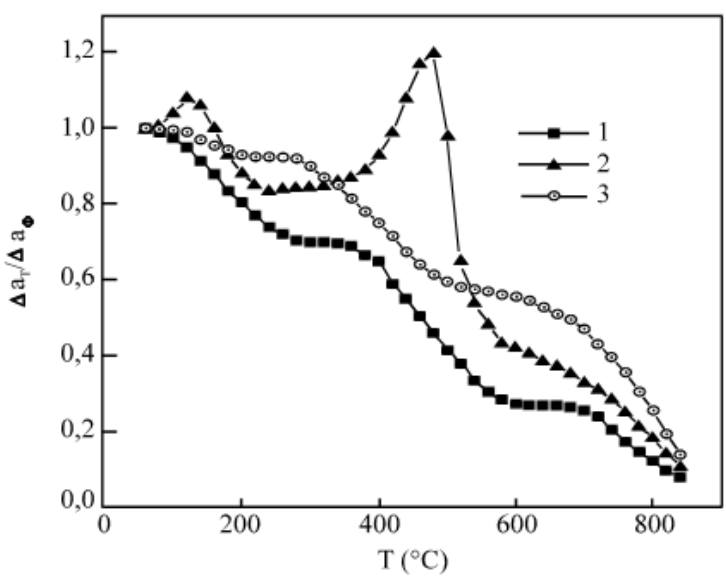

Fig. 2: Lattice Constant Recovery at Isochronal 15 min Annealing of Implanted Silicon. $\Delta \mathrm{a}_{\Phi-}$ Change of Lattice Constant after Ion Implantation with Fluence $\Phi ; \Delta \mathrm{a}_{\mathrm{T}}$-Change of Lattice Constant after Ion Implantation and Annealing at Temperature T.1)-Si:P, $\rho_{0}=0.5$ $\Omega \mathrm{cm}$; (2)-Si:B, $\rho_{0}=0.005 \Omega \mathrm{cm}$; (3)-Si:B, $\rho_{0}$ $=0.005 \Omega \mathrm{cm}$, Annealing with Electron Illumination

The stage at $700-900^{\circ} \mathrm{C}$ is characteristic of silicon implanted with $\mathrm{B}^{+}$ions only and may be explained by annealing of radiation defect complexes which include boron atoms.

In silicon heavily doped with boron the stages of "inverse" annealing at temperatures of 100-120 and $380-500^{\circ} \mathrm{C}$ are superimposed to the curves of the lattice constant recovery (Fig. 2, curve 2). However, for the same samples, the curves of isochronal annealing with electron illumination have not the "inverse" stages (curve 3).

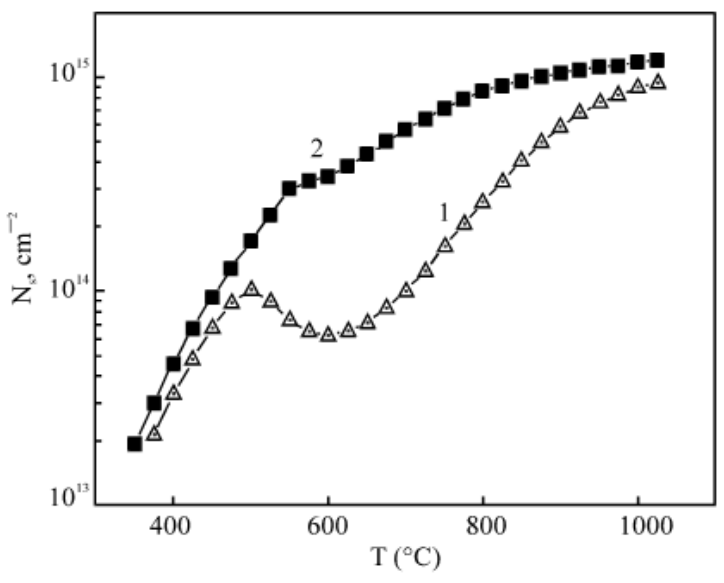

Fig. 3: Curves of Electrical Activation of Ionimplanted Boron in Silicon by Isochronal Annealing without (1) and with Electron Illumination (2)

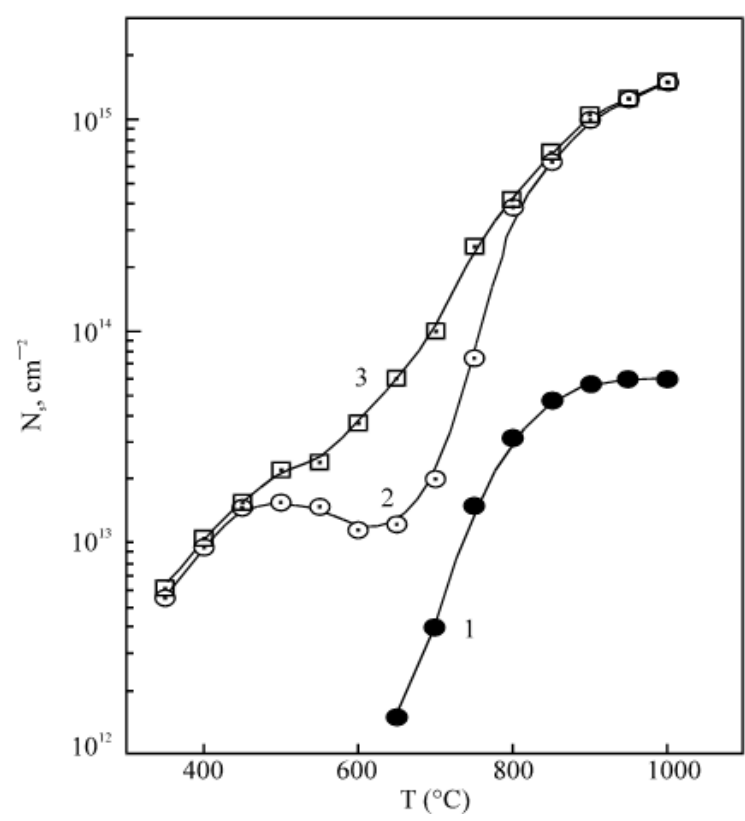

Fig. 4: Curves of Electrical Activation of Ionimplanted Phosphorus in Silicon by Isochronal Annealing: (1)- $\Phi_{\mathrm{P}}=6.3 \times 10^{13} \mathrm{~cm}^{-2} ;(2)-\Phi_{\mathrm{P}}=$ $1.5 \times 10^{15} \mathrm{~cm}^{-2}$, then Annealing and $\Phi_{\mathrm{Si}}=1$ $\times 10^{14} \mathrm{~cm}^{-2}$; (3)-the same as (2) but Annealing with Electron Illumination

Figure 3 presents curves of electrical activation of implanted boron in silicon (the dependence of the sheer concentration of the charge carriers NS on the temperature of isochronal $15 \mathrm{~min}$ annealing) without (curve 1) and with (curve 2) simultaneous $10 \mathrm{keV}$ electron irradiation with a current density of $5 \mu \mathrm{A} \mathrm{cm}^{-2}$. The boron activation curve contains a stage of "reverse" annealing (curve 1). 


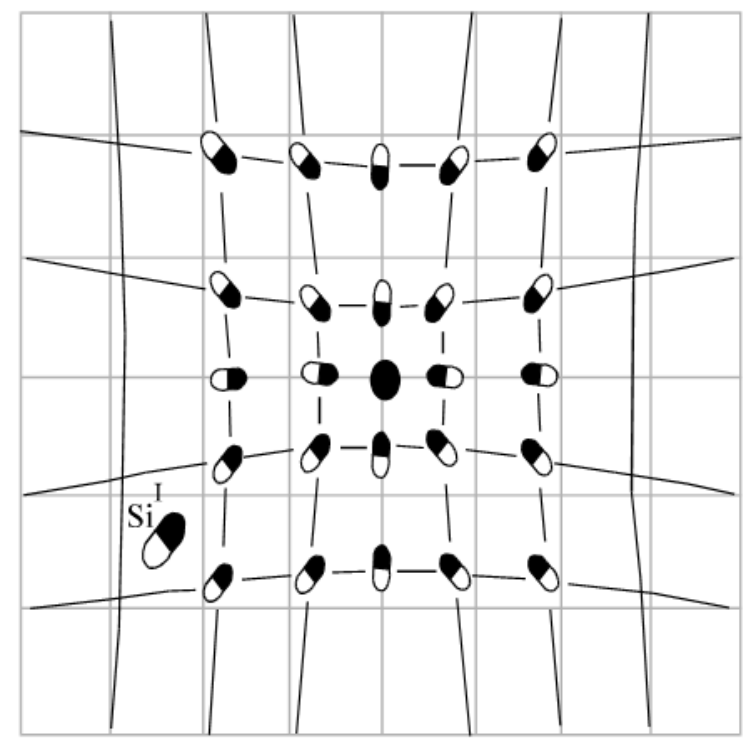

Fig. 5: Motion of Interstitial Si Atom under Elastic Deformation Field

In the case of boron activation during thermal treatment with simultaneous electron illumination only a bend is observed in the activation curve (curve 2).

The electrical activation of implanted phosphorus is shown in Fig. 4. The phosphorus activation curves do not contain stages of "reverse" annealing usually (curve 1). At the high P fluences $\left(>6 \times 10^{14} \mathrm{~cm}^{-2}\right)$ amorphization of silicon layer takes place and electrical activation of the impurity during the annealing occurs simultaneously with the crystallization process. However, "reverse" annealing was present (curve 2) if the samples have been implanted with a high influence of phosphorus ions and then subsequently annealed to recrystallize amorphous layer and irradiated with $\mathrm{Si}^{+}$ ions to generate the radiation defects. But the "reverse" stage is absent if the thermal treatment is carried out with simultaneous electron irradiation (curve3).

The great increase of lattice constant resulting from implantation (Fig. 1) in heavily boron doped silicon as compared to n-type silicon crystals cannot be explained by the higher concentration of radiation defects. It has been ascertained in backscattering studies ${ }^{[10]}$ that the presence of boron atoms does not influence the rate of introduction of stable defects in silicon crystals. The boron atoms participate probably in the formation of thermally stable complexes (stage at $700-900^{\circ} \mathrm{C}$ ) during annealing. We suggest that the additional increase of lattice constant in silicon heavily doped with boron may be connected with the replacement of boron atoms from the nodes by self-interstitial generated during implantation. The boron atoms positioned at the nodes compress the lattice of silicon. The covalent radius of $\mathrm{Si}$ atom is $0.1175 \mathrm{~nm}$, while for B-0.08 nm. The lattice constant is growing as the boron atoms are displaced from the nodes. The concentrations of replaced B atoms may be estimated from the differences in $\Delta \mathrm{a}$ and in the covalent radii of $\mathrm{Si}$ and $\mathrm{B}$ atoms. At the $\mathrm{B}^{+}$ion fluence of $2 \times 10^{14} \mathrm{~cm}^{-2}$ the concentrations of displaced boron atoms from the nodes are estimated to be $8 \times 10^{17}$, $5.9 \times 10^{18}$ and $2 \times 10^{19} \mathrm{~cm}^{-3}$ at ion current densities of 0.5 , 0.2 and $0.05 \mu \mathrm{A} \mathrm{cm}^{-2}$, accordingly. The initial boron concentration in silicon samples was $4 \times 10^{19} \mathrm{~cm}^{-2}$. With decreasing of ion current density the quantity of boron displacement increases. This effect is joined with a level of ionization in implanted layer. It correlates with results on defects annealing (Fig. 2). The stages of "inverse" annealing are due to substitution of boron atoms in the lattice nodes by interstitial $\mathrm{Si}$ atoms. The temperatures of "inverse" annealing coincide with the annealing temperatures of interstitial complexes Si$\mathrm{P} 6^{[11]}$ and $\mathrm{Si}-\mathrm{B} 3^{[12]}$. With increasing ionization level in the layer (electron illumination) the substitution process is suppressed (Fig. 2, curve 3).

Concerning activation process, it was shown using channeling method and electrical measurements ${ }^{[13]}$ that the "reverse" stage in the activation curves is obliged to the boron displacement from the lattice nodes by interstitial $\mathrm{Si}$ atoms generated during the annealing of the interstitial complexes. At high concentrations of impurity and radiation defects, this process is seen in the curves of electrical activation of inserting phosphorus too. However, in the cases of boron and phosphorus the process of Watkins substitution is suppressed at high level of ionization in implanted layer. It follows from both X-ray diffraction and electrical investigations.

According to the ideas developed in the work ${ }^{[14]}$, the Watkins substitution is determined by the movement of interstitial $\mathrm{Si}$ atoms toward impurity atoms in the elastic strain field generated by these impurities due to misfit of the covalent radii of impurity and lattice atoms. The lattice atoms displaced from the equilibrium positions in the first coordinate sphere relative to the impurity atom and to a less extent in the subsequent spheres (fading) is polarized and generate an electric dipole moment. Under the influence of the dipoles of the displaced atoms, the interstitial $\mathrm{Si}$ atoms obtain inductive dipole moments. Since the magnitude of the dipole moment sharply increases towards the center of the deformation, the interstitial $\mathrm{Si}$ atom moves to a compression (dilatation) center. This movement is schematically presented in Fig. 5. But what is the reason of the suppression of the substitution process when the level of ionization in the implanted layers is high? First, it must be considered from the point of view of charge states of interacting species. Boron and phosphorus impurities being located at the Si lattice nodes have opposite charge states. If the self-interstitial capture any type of nonequilibrium charge carriers (electrons or holes) it cannot suppress substitution process in the equal degree for both impurities (boron and phosphorus). The same results for $\mathrm{B}$ and $\mathrm{P}$ atoms having the opposite charge states in the nodes, 
demonstrates that the reason of the substitution process suppression is not conditioned by charge states of the impurities and self-interstitials. We believe that at the highest level of ionization, the nonequilibrium electrons and hole screen the electric dipoles in the deformed sphere with the result that the motion of $\mathrm{Si}$ atoms in the elastic strain field generated by the node-situated atom is suppressed.

\section{CONCLUSION}

The presented results of the X-ray diffraction and electrical investigations show that the efficiency of displacement of boron atoms from the lattice nodes by self-interstitial depends upon a level of ionization in implanted silicon layers. The substitution process may be suppressed at the condition that the effective ion current density must be more than $0.5 \mu \mathrm{A} \mathrm{cm} \mathrm{cm}^{-2}$ during $100 \mathrm{keV} \mathrm{B}^{+}$scanning beam implantation. During annealing the process of boron substitution may be suppressed using low-energy electron irradiation with an electron current density of $5 \mu \mathrm{A} \mathrm{cm}$.

\section{REFERENCES}

1. Tamura, M., T. Ando and K. Ohyu, 1991. MeVion-induced damage in $\mathrm{Si}$ and its annealing. Nucl. Instr. and Methods in Phys. Res. B, 59 (7): 572583.

2. Simpson, T.W., R.D. Goldberg and I.V. Mitchell, 1995. Suppression of dislocation formation in silicon by carbon implantation. Appl. Phys. Lett., 67(19): 2857-2859.

3. Bonafos, C., A. Claverie, D. Alquier, C. Bergaud, A. Martinez, L. Laanab and D. Mathiot, 1997. The effect of the boron doping level of the thermal behavior of end-of-range defects in silicon. Appl. Phys. Lett., 71(3): 365-367.

4. Watkins, G.D., 1975.Defects in irradiated silicon: EPR and electron-nuclear double resonance of interstitial boron. Phys. Rev., 12(12): 5824-839.
5. Liefting, J.R., R.J. Schreutelkamp, J. Vanhellemont, W. Vandervorst, K. Maex, J.S. Custer and F.W. Saris, 1993. Electrically active, ion implanted boron at the solubility limit in silicon. Appl. Phys. Lett., 63(8): 1134-1136.

6. Van der Pauw, L.J., 1958. A method of measuring specific resistivity and Hall effect of the discs of arbitrary shape. Philips Res. Reps., 13(1): 1-9.

7. Berezhnov, N.I., V.F. Stelmakh and A.R. Chelyadinskii, 1983. Interstitial type defects in ion implanted silicon. Phys. Stat. Sol. A, 78 (2) K121K125.

8. Jung, W. and G.S. Newel, 1963. Spin 1 center in neutron irradiated silicon. Phys. Rev., 132(2): 648662 .

9. Araika, O.J., A.R. Chelyadinskii, V.A. Dravin, Yu.R. Sprung-Belevich and V.P. Tolstikh, 1993. Radiation defects and electrical properties of silicon layers containing $\mathrm{Sb}$ and As implanted with $\mathrm{Si}^{+}$ions. Nucl. Instr. and Methods in Phys. Res. B, 73 (4): 503-506.

10. Hirvonen, J.K. and F.H. Eisen, 1971. A lattice disorder in ion implanted boron-doped silicon. Appl. Phys. Lett., 19(1): 14-16.

11. Lee, Y.H., N.N. Gerasimenko and J.W. Corbett, 1976. EPR study of neutron-irradiated silicon: A positive charge state of the $\langle 100\rangle$ split diinterstitial. Phys. Rev. B, 14 (12): 4506-4510.

12. Brower, K., 1976. EPR of $\langle 100\rangle \mathrm{Si}$ interstitial complex in irradiated silicon. Phys. Rev. B, 14 (3): 872-883.

13. North, J.C. and W.M. Gibson, 1970. Channeling study of boron-implanted silicon. Appl. Phys. Lett., 16(3): 126-129.

14. Berezhnov, N.I., A.R. Chelyadinskii, M. Jadan and Yu.R. Sprung-Belevich, 1993. On the problem of Watkins substitution and migration of silicon atoms in silicon. Nucl. Instr. And Methods of Phys. Res. B, 73 (3): 357-363. 